\title{
Server-Uncertain Spectrum Trading in Cognitive Radio Networks: A Queueing-Theoretic Modeling Approach
}

\author{
Lixia LIU*, Gang HU†, Ming XU†, Yuxing PENG* \\ $*$ National Key Laboratory for Parallel and Distributed Processing, $\nmid$ Department of Network Engineering \\ College of Computer, National University of Defense Technology \\ Changsha, China 410073 \\ Email: 5460liulixia@126.com, golfhg@vip.sohu.net, xuming@nudt.edu.cn, pengyuxing1963@yahoo.com.cn
}

\begin{abstract}
Spectrum trading is the promising method to improve spectrum efficiency from the perspective of economics. In this paper we propose a queueing-theory based spectrum trading model, where the primary user plays the server role providing spectrum to the secondary user who acts as the customer. The most significant challenge is how to optimize the spectrum trading model considering the server uncertainty which includes service state, service time, service area, service content and service price. We design a STACP queueing model according to the server attributes, so that the secondary user can choose the right queue quickly and reasonably according to its demand. Moreover, we further analyze the optimizing strategies for STACP model which can maximize the profit of the primary user and minimize the service cost of the secondary user. The simulation results demonstrate the analysis results.
\end{abstract}

Index Terms-Dynamic spectrum access; cognitive radio; spectrum trading; queueing; server uncertainty; average service rate

\section{INTRODUCTION}

Dynamic spectrum access [1] has attracted wide and intensive attention as the promising method to solve the spectrum under-utilization problem and satisfy the rapid-developing demand for spectrum in recent years.

From the technical perspective, the cognitive radio proposed by Mitola in [2] can sense the radio environment, find the unused spectrum and access the spectrum opportunistically without interfering the primary user (PU, the licensed user). It relates to many complex operations, such as spectrum sensing, power control, spectrum allocation, spectrum handoff and so on. Although the secondary user (SU, the unlicensed user) can share the spectrum resource for free, its communication quality can't be guaranteed because it has to quit from the spectrum immediately once PU needs the spectrum. PU will not take part in the spectrum sharing process actively because nothing can motivate it to cooperate with SU. So it just cares its own business.

From the economic perspective, PU would like to lease or sell the unused spectrum to SU via spectrum trading so that it can gain some profit to counterbalance the cost of getting the spectrum license from the spectrum provider. SU only has to pay something for the spectrum access chance and don't need to worry about the interference to PU and conflict with other SU. Spectrum trading, which can improve the spectrum efficiency obviously, is an advisable method especially for the users who have high demands to the spectrum quality and don't consider the payment problem. Some economic models, such as auction and game theory, have been investigated [3] [4]. Price has been paid much attention to as well as strategy.

Qian et al. proposed an agent-based spectrum trading model [5] and tried to maximize the profit of the agent as well as to enhance the satisfaction of secondary users. Mwangoka proposed a multiple-dimension auctioning mechanism through a broker [6] and discussed two trading negotiation protocols, merchant and auction. The broker's objective is to maximize its revenue. Duan proposed a cognitive mobile virtual network operator(C-MVNO) [7] which serves as the interface between the spectrum owner and the secondary end-users. In the practical application, the primary user and the secondary user both want no agent, broker or operator to participate in the spectrum trading if they can do it by themselves. It is the basic idea that no one would like to share the profit with others.

Tan modeled a non-cooperative pricing game [8] using the profit as the payoff and studied short-term price war and long-term price war. Price is the crucial problem of spectrum trading, but it is not enough to concentrate on how to formulate an optimal pricing strategy to maximize the revenue from the perspective of spectrum provider without taking the customer behavior into consideration. In the real business world, the demand also affects the spectrum market as well as the supply.

In this paper we consider a queueing theory based spectrum trading model, where PU plays the server role and SU acts as the customer without any broker or agent. Considering the server uncertainty, we construct the virtual queues according to service state, service time, service area, service content and service price. The actual queues are decided by the primary users. The customer can choose the right queue quickly and reasonably on the basis of its demands. By designing the optimization measures for the spectrum trading model, this paper concerns less idle probability and more profit for the server under its service intensity and less service cost for the customer. 
The remainder of this paper is organized as follows. Section II describes the attributes of server uncertainty and the customer behavior. Section III provides a brief introduction to the STACP queueing system after analyzing the relationship between servers, and the related optimization measures are presented in Section IV, followed by simulations drawn in Section V. A simple conclusion will be given in the last section.

\section{Problem Description}

\section{A. Server Uncertainty}

The server in spectrum trading model is different from the one in ordinary queueing system because some attributes, such as service state, service time, service area, service content and service price, are uncertain due to the special spectrum service.

1) Service State: During spectrum trading, the spectrum provider would rather keep the busy state than be idle for getting more profit unless it has to use the spectrum itself. So three service states of the server should be considered as shown in Fig.1: busy, idle and leave.

$S_{s(i)}(t) \triangleq$ service state of $s(i)$ at time $t=\left\{\begin{array}{c}0, \text { idle } \\ 1, \text { busy } \\ -1, \text { leave }\end{array}\right.$

$S_{s(i)}(t)=0$ means server $i$ is idle at time $t$ and can provide service, $S_{s(i)}(t)=1$ means server $i$ is providing service for customer at time $t$, and $S_{s(i)}(t)=-1$ means server $i$ has to deal with its own traffic and can't provide service. The three states can transform to each other.

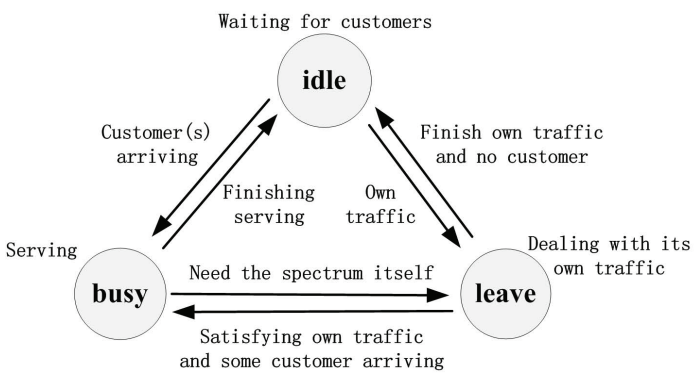

Fig. 1. The states transform of primary user

2) Service Time: The service time in spectrum trading is determined by not only the server but also the customer. Since the primary user may quit the spectrum trading at any moment, the provided service time of the primary user may also be uncertain. We define the demanded service time of the customer $j$ as:

$$
T_{c(j) \_d}(t) \triangleq \text { demanded service time of } c(j) \text { at time } t
$$

which is decided by the customer's traffic. Also we denote the provided service time by the server $i$ as:

$$
T_{s(i) \_p}(t) \triangleq \text { provided service time by } s(i) \text { at time } t
$$

If the provided service time is long enough to satisfy the customer's demand, the actual service time equals to the demanded service time. On the contrary, if the provided service time is not long enough, the actual service time equals to the provide service time. The service time when the server $i$ serves for the customer $j$ can be denoted as:

$$
\begin{aligned}
& T_{[i, j]}(t) \triangleq \text { service time of } s(i) \text { for } c(j) \text { at time } t \\
& =\min \left(T_{c(j) \_d}(t), T_{s(i) \_p}(t)\right)
\end{aligned}
$$

3) Service Area: For the authorization reason, each primary user can only serve for the customers in a given area. Those customers, who aren't located in the service area of $s_{i}$, can't enjoy the service of $s_{i}$. In other words, the customers that arrive at the queueing system can't choose the server randomly. We also give a definition of the service area as:

$$
A_{s(i)}(t) \triangleq \text { service area served by } s(i) \text { at time } t
$$

4) Service Content: Since the primary users have different spectrum resources (frequency, bandwidth and so on), the service contents from all the servers must be different, too. The service content can be denoted as:

$$
C_{s(i)}(t) \triangleq \text { service content served by s(i) at time } t
$$

5) Service Price: Difference prices will be regulated according to different service quality (permitted transmission power and so on) of spectrum resources. What's more, the spectrum price should vary with the market change according to the basic economic theory. If the supply is more than the demand, the price will be lower. On the contrary, the price should be higher. We define $P_{s(i)}$ as the unit service price per unit time and unit bandwidth.

$$
P_{s(i)}(t) \triangleq \text { unit service price of } s(i) \text { at time } t
$$

\section{B. Customer Behavior}

1) Sojourn Time: The sojourn time of customer can be discussed as the total service time of server because the sojourn time is the sum of service time and waiting time.

$$
\begin{aligned}
T_{w(j)} & (t) \triangleq \text { waiting time of } c(j) \text { at time } t \\
& =\sum_{\substack{j=1 \\
j=1}} T_{[i, x]}\left(t^{\prime}\right) \\
& =\sum_{x=1}^{j-1} \min \left(T_{c(x) \_d}\left(t^{\prime}\right), T_{s(i) \_p}\left(t^{\prime}\right)\right)
\end{aligned}
$$

where $t^{\prime}=t-(j-x) \times \tau$. After serving customer $j$, the server $i$ can provide the service time at time $t+1$ as:

$$
T_{s(i) \_p}(t+1)=\max \left(T_{s(i) \_p}(t)-T_{[i, j]}(t), 0\right)
$$

If $T_{s(i) \_p}(t+1)=0$, it means that the server $i$ can't provide service at time $t+1$ and the customer has to choose another 
queue. The sojourn time can be denoted as:

$$
\begin{aligned}
T_{s o j(j)} & \triangleq \text { sojourn time of } c(j) \\
= & T_{[i, j]}(t)+T_{w(j)}(t) \\
= & \min \left(T_{c(j) \_d}(t), T_{s(i) \_p}(t)\right)+\sum_{k=1}^{j-1} T_{[i, k]}\left(t^{\prime}\right) \\
& =\min \left(T_{c(j) \_d}(t), T_{s(i) \_p}(t)\right) \\
& +\sum_{k=1}^{j-1} \min \left(T_{c(k) \_d}\left(t^{\prime}\right), T_{s(i) \_p}\left(t^{\prime}\right)\right)
\end{aligned}
$$

where $t^{\prime}=t-(j-k) \times \tau$.

2) Queue Choice: The customer in the spectrum trading is different from the ones in the ordinary queueing system when they make the queue choice. The latter will select the empty queue or the queue with the fewer customers. While the former will select the queue which is more fit for itself after considering the service time, service price and so on comprehensively. If the latter determines the queue according to the queue size, the former does it according to its demand.

\section{Service Platform}

We assume that there exists a service platform on which the primary users and the secondary users can finish the spectrum trading. In the practical scenario the base station or some resource center both can be the ideal platform provider.

\section{QUeUeInG MODEL}

Since there are so many differences between the ordinary queueing system and spectrum trading, it is necessary to consider a special queueing system for spectrum trading considering the server uncertainty and the customer behavior.

\section{A. Relationship Between Servers}

In the ordinary queueing system, there is no difference between any two servers because they provide the same service. While in the spectrum trading system, a server may be different from any other server for its spectrum resource. The relationship between any two servers is complex.

1) Independent: Since the spectrum resource of a server is different from other spectrum resource, the service of the server is also independent with other servers' services. Each server can determine the service attributes (such as state, time, area, content, price) by itself. Each server can serve for the customers independently.

2) Competition: All the servers would like to contend for more customers and maximize its own profit. So the competition for the customers is inevitable between the servers. To attract more customers, the server will take actions such as decreasing the spectrum price or increasing the service time.

3) Cooperation: When some server has too many customers and can't provide timely service, it may introduce some of its customers to other servers who can satisfy them as well. For this case the server shows the cooperation desire with other servers. It will happen only when the server hasn't the ability to deal with all the business.

\section{B. STACP Queueing Model}

Based on the queueing theory and attributes of server, a spectrum trading model-STACP (State, Time, Area, Content and Price) can be taken into account. In Fig.2 the queue is divided into the virtual queue and the actual queue so that the customers can find the right queue quickly. The number of the virtual queue can be denoted as:

$$
N_{v q}=n_{s} \times n_{t} \times n_{a} \times n_{c} \times n_{p}
$$

Where $n_{s}$ is the number of service state, $n_{t}$ is the number of service time, $n_{a}$ is the number of service area, $n_{c}$ is the number of service content and $n_{p}$ is the number of service price. As shown in Fig.2, $n_{s}=3$ and $n_{t}=n_{a}=n_{c}=n_{p}=2$, then $N_{v q}=3 * 2 * 2 * 2 * 2=48$. If needed, each attribute can be divided in detail. By assuming that each server can serve for only one queue, we can get that the actual queue number equals to the serve number $n$.

$$
N_{a q}=\text { the server number } n
$$

Each queue in the actual queue is corresponding to only one of the virtual queue. And two or more queues in the actual queue may be corresponding to the same queue of the virtual queue. In Fig.2 queue 2 and queue 3 of the actual queue are both corresponding to $S_{1} T_{1} A_{1} C_{2} P_{2}$ of the virtual queue. There is no affiliation relationship between the virtual queue and the actual queue.

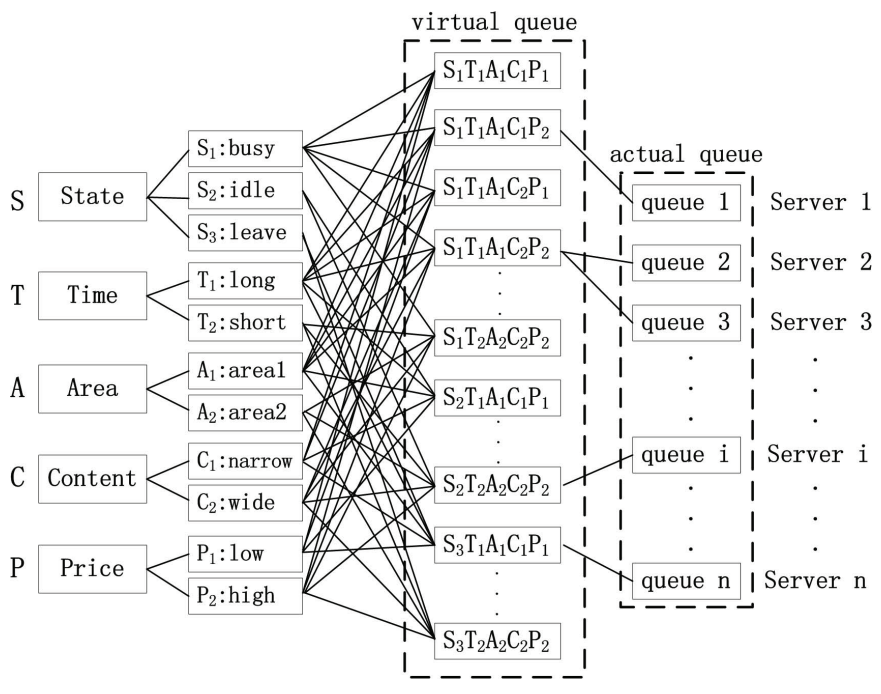

Fig. 2. The virtual queue and the actual queue

Before the customer makes the queue choice, it does the matching process first. It matches its demands to the virtual queue and finds the corresponding queue in the actual queue. If there is no completely matching queue, some matching strategies should be considered:

- Most Attributes Matching. The customer chooses the server whose service can satisfy most of its demands. 
- Attribute Priority Matching.The customer chooses the server whose service can satisfy its important demands.

\section{Optimal StRATEgY For Queueing Model}

To achieve better performance, it is not enough to design the queueing model for spectrum trading. Some optimal strategies should be used when running this queueing model.

\section{A. Idle Percentage of Server}

By assuming that there are $w$ customers in the service queue of server $i$ and the total time is $T_{\text {total }}$, the idle percentage of server $i$ can be defined as:

Definition 1 (Idle Percentage of Server $i$ ) The idle percentage of server $i \phi(i)$ shows the percentage of the idle time to the total time. It can be denoted as:

$$
\begin{aligned}
& \varphi(i) \triangleq \text { the idle percentage of server } \mathrm{i} \\
& =\frac{T_{\text {total }}-T_{\text {service }}(i)}{T_{\text {total }}} \times 100 \% \\
& =\frac{T_{\text {total }}-\sum_{j=1}^{w} T_{[i, j]}(t)}{T_{t o t a l}} \times 100 \% \\
& =\frac{T_{\text {total }}-\sum_{j=1}^{w} \min \left(T_{c(j) \_d}(t), T_{s(i) \_p}(t)\right)}{T_{\text {total }}} \times 100 \%
\end{aligned}
$$

Then we can get the idle percentage of queueing system on the basis of $\phi(i)$ :

$$
\begin{aligned}
& \Phi=\frac{\sum_{i=1}^{n}\left(T_{\text {total }}-T_{\text {service }}(i)\right)}{T_{\text {total }} \times n} \times 100 \%
\end{aligned}
$$

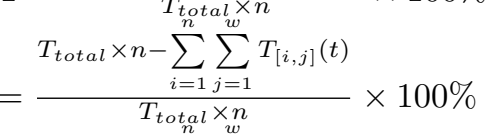

$$
\begin{aligned}
& =\frac{T_{\text {total }} \times n-\sum_{i=1}^{n} \sum_{j=1}^{w} \min \left(T_{c(j) \_d}(t), T_{s(i)} \_^{p}(t)\right)}{T_{\text {total }} \times n} \times 100 \%
\end{aligned}
$$

If the service state is idle, the server is neither busy in its own traffic nor providing service for customers. The idle state means not only the waste of spectrum access opportunity but also the profit loss. The queueing model for spectrum trading prefers to less idle percentage of servers and needn't consider about the service intensity because the service of spectrum providing doesn't make the server tired. So one of our target is to minimize the overall idle percentage $\Phi$.

Minimizing $\Phi$ equals to maximize the service profit or the spectrum efficiency. To reduce the idle percentage, we can: 1) reduce the server number, 2) increase the customer number, 3) increase the demanded service time. Although the service rate can affect the busy time of the server in the ordinary queueing theory, it is meaningless for the spectrum trading because the service that the server provides to the customer is the spectrum access time.

1) Server Number: Generally speaking, the server number $n$ can't be too large or too small. If there are too many servers, some of them will have no customer and the idle state reduces the spectrum efficiency. On the contrary if there are too few servers, many customers have to wait for a long time before they are served. So it is important to keep an appropriate server number. It has mentioned above that the server has three states: busy, idle and leave, which is decided by its customers as well as its own spectrum demand. Therefore if we want to adjust the server number, adjusting its customer number and its demand are two convenient ways. What's more, the server number should not be changeless. The change of customer number leads to the corresponding adjustment of server number.

2) Customer Number: The customers arrive at the queues once they need to use the spectrum. From the perspective of one server, increasing the customer number can reduce the idle percentage of server. But it is noteworthy that the customer can enter the queue only when it demands for the spectrum resource. So we can not increase the customer number randomly. If considering the overall system and the certain customer number, the feasible way to reduce the idle percentage is to make the customers well-distributed as possible. In other words, the customers in a too crowded queue can be scheduled to other empty queue where the server can also satisfy its demand.

3) Demanded Service Time: If the demanded service time $T_{c(j) \_d}(t)$ is long enough, the probability that the server state is idle is small. The idle percentage $\phi$ follows inverse ratio to the demanded service time $T_{c(j)}{ }_{d}(t)$. While we don't need to consider the provided service time because if $T_{s(i) \_p}(t)$ is large, the server can provide more time to serve the customer and if $T_{s(i) \_p}(t)$ is small, that means the server spends more time on its traffic.

\section{B. Service Cost of Customer}

In this paper we also consider about the service cost of the customer which includes two parts: the waiting loss and service price. For any customer $j$, the service cost can be denoted as:

$$
\begin{aligned}
& \theta(i, j) \triangleq \text { the service cost of customer } j \\
& =\text { the waiting loss }+ \text { the service price } \\
& =\alpha \times T_{w(j)}(t)+P_{s(i)}(t) \\
& =\alpha \times \sum_{x=1}^{j-1} \min \left(T_{c(x) \_d}\left(t^{\prime}\right), T_{s(i) \_p}\left(t^{\prime}\right)\right)+P_{s(i)}(t)
\end{aligned}
$$

where $\alpha$ is the factor which can transform the waiting time to the waiting loss and it denotes the waiting loss per unit time.

Considering all the $m$ customers, the overall service cost can be denoted as:

$$
\begin{aligned}
& \Theta=\sum_{k=1}^{m} \theta(i, k) \\
& =\sum_{k=1}^{m}\left(\alpha \times T_{w(k)}(t)+P_{s(i)}(t)\right) \\
& =\sum_{k=1}^{m}\left(\alpha \times \sum_{x=1}^{j-1} \min \left(T_{c(x) \_d}\left(t^{\prime}\right), T_{s(i) \_p}\left(t^{\prime}\right)\right)+P_{s(i)}(t)\right)
\end{aligned}
$$

where customer $k$ has to wait for $j-1$ customers before it can be served in queue $i$.

It is obvious that our another object is to minimize the overall service cost $\Theta$.

To minimize the service cost, we can: 1$)$ reduce the waiting loss, 2) reduce the service price. 
1) Waiting Loss: The queue size should not be used to measure the waiting loss because the service times of all the customers may be different. Instead the total waiting time can measure the waiting loss. In the above description we formulate the waiting loss as $\alpha \times T_{w(j)}(t)$. Since any customer has no right to move other customers away, it can't make the waiting time to become shorter unless it has higher priority and can jump a queue. However it has to pay with higher service price, which will increase the service cost.

Therefore the most feasible way is to choose another queue which has shorter waiting time and also can satisfy its demand.

2) Service Price: The service price is decided by the server. It seems that the customer can't reduce the service price. Actually if the customers all choose the queue which has the lower price, it will force the server to reduce the service price for attracting more customers and reducing the idle percentage. So the customer can reduce the service price by choosing the queue which has lower price.

Since the idle percentage of PU and the service cost of SU are both related to the customer number, the service price and so on, we must find the equilibrium point. For example, PU prefers more customers for more profit and SU prefers fewer customers for less waiting loss. So we must find a customer number to satisfy both of them.

\section{Simulation AND Discussion}

To demonstrate the analysis about the spectrum trading model, we design the detailed simulations. By the assumption that the arrival rate of the customers follows the exponential distribution, it generates the demands (service time, service area, service content and service Price) randomly for each customer. The simulation results will show how the server number, the customer number and the demanded service time affect the idle percentage. The simulations also demonstrate the performance advantages of the STACP queueing model comparing with the queueing in turns.

Fig. 3 shows the relationship of the idle percentage and the server number. The arrival interval of the customers is 2 . The server number varies within [1,10]. It can be observed in Fig. 3 that the idle percentage increases with the increasing of the server number as we described in section IV(A). Fig.3 addresses that it can improve the spectrum efficiency by reducing the server number if the remained servers have the ability to deal with the business. All the results in Fig. 3 are average values after running 100 times.

Fig. 4 shows the relationship of the idle percentage and the customer number. The server number $n$ is 5 . The arrival interval $\mu$ varies in $[0.1,4]$, and the customer number decreases from 501.3 to 13.3 . The idle percentage changes from $0.75 \%$ to $97.72 \%$. This means that the spectrum efficiency can be improved if more customers are permitted to use the spectrum. All the results in Fig.4 are average values after running 100 times.

In Fig. 5 we assume that the longer demanded service time is five times of the shorter one. The shorter demanded service time varies in [1.5,5] and the longer demanded service time

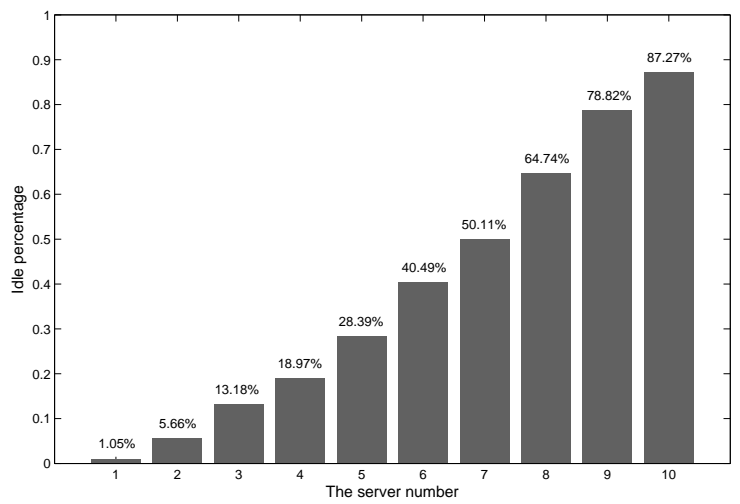

Fig. 3. Idle percentage and server number



Fig. 4. Idle percentage and customer number

varies in $[7.5,25]$ correspondingly. The arrival interval of customers is 2 , and the service number is 5 . It is apparent that the idle percentage reduces with the increasing of the demanded service time. It improves that the longer the demanded service time is, the more time the servers spend on service and so the higher spectrum efficiency it can achieve.

In Fig.6 we show the advantage of STACP queueing model comparing with queueing in turns in terms of the idle percentage. This simulation runs for 100 times and it is obvious that the idle percentage of STACP queueing is less than that of queueing in turns. The average idle percentage of STACP queueing is near $30 \%$, and that of queueing in turns is about $60 \%$. That is to say the spectrum efficiency can be improved about $30 \%$ by STACP queueing model.

In Fig. 7 we set the shorter demanded service time is 2 and the longer demanded service time varies according to the times as X-axis. We can observe from Fig.7 that the idle percentage decreases with the increasing of the times, which suggests that we can improve the spectrum efficiency by enlarging the longer demanded service time. However we must notice that the performance change is not so obvious when the times is larger than 10 . So it is unnecessary to larger the times 
endlessly.

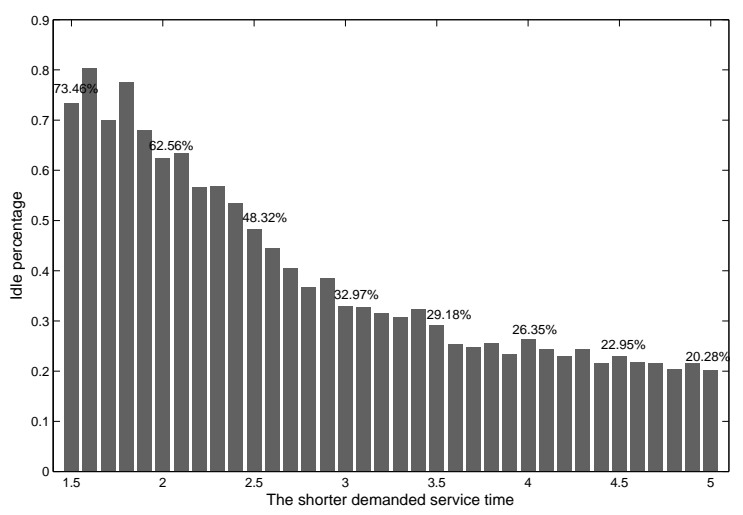

Fig. 5. Idle percentage and the demanded service time

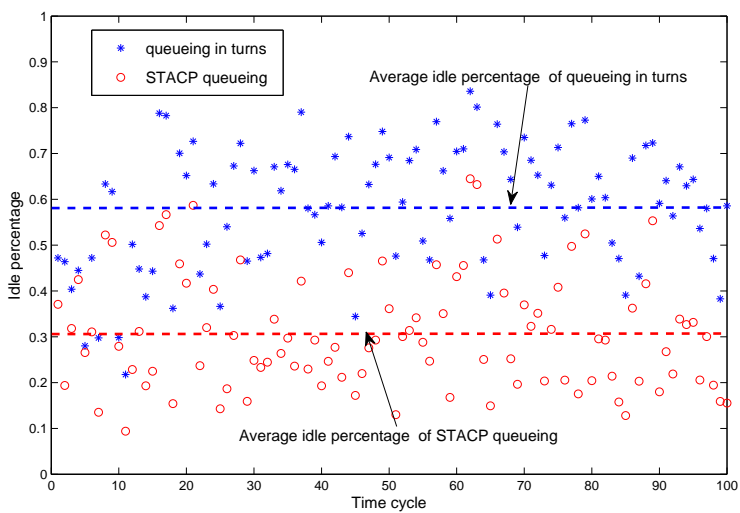

Fig. 6. The comparison about idle percentage



Fig. 7. Times of the shorter demanded service time

Due to the page limitation, more simulations about the service cost of the customer and so on will be given in our extension work.

\section{CONCLUSION}

Spectrum trading is a promising method to improve the spectrum efficiency and satisfy the increasing spectrum demand. The active participation of the primary users can not only improve their profit, but also lighten the secondary users' burden so that they needn't spend lots of time on spectrum sensing, allocation and so on. To maximize the profit of PU and minimize the cost of SU, we address a spectrum trading model based on queueing theory, where PU acts as the server and SU acts as the customer. Considering the server uncertainty, we propose the virtual queue based on the service attributes and the corresponding actual queue decided by the server. The customers can find the proper queue quickly according to its demand. Furthermore we analyze the optimal measures in terms of reducing the idle percentage of PU and reducing the service cost of SU. The simulation result and discussion show that this trading model can improve the spectrum efficiency as we expected.

\section{ACKNOWLEDGMENT}

This research work is supported by National Basic Research Program of China under Grant No.2011CB302601, National high technology research and development program No.2011AA01A202, National Natural Science Foundation of China under Grant No.61070211, No. 60903223, and Foundation of Ministry of Education of China under Grant No.20104307110004, No.20114307120003.

\section{REFERENCES}

[1] A. A. Qing Zhao, "A survey of dynamic spectrum access: Signal processing and network perspectives," in Proc. of IEEE International Conference on Acoustics, Speech, and Signal Processing, 2007, pp. 1349-1352.

[2] J. J.Mitola, Q.Gerald, "Cognitive radios: Making software radios more personal." in IEEE Personal Communications,Vol.6, No.4, 1999, pp. 1318.

[3] K. M. V. Rodriguez and R. Tafazolli, "Auction driven dynamic spectrum allocation: Optimal bidding, pricing and service priorities for multi-rate, multi-class cdma," in in Proc. IEEE PIMRC05, vol. 3, Sept, 2005, pp. 1850-1854.

[4] D. Niyato and E. Hossain, "A game-theoretic approach to competitive spectrum sharing in cognitive radio networks," in in Proc. IEEE WCNC07, 2007, pp. 16-20.

[5] L. G. X. G. T. C. X. T. X. W. Liang Qian, Feng Ye and M. Guizani, "Spectrum trading in cognitive radio networks:an agent-based model under demand uncertainty," IEEE Transactions on Communications, pp. 3192-3203, 2011.

[6] P. M. Joseph W.Mwangoka and J. Rodriguez, "Broker based secondary spectrum trading," in 2011 6th International ICST Conference on Cognitive Radio Oriented Wireless Networks and Communications(CROWNCOM), 2011, pp. 186-190.

[7] B. S. Lingjie Duan, Jianwei Huang, "Investment and pricing with spectrum uncertainty: A cognitive operator's perspective," IEEE Trans.Mob.Comput., pp. 1590-1604, 2011.

[8] K. Yi Tan, Shamil Sengupta, "Competitive spectrum trading in dynamic spectrum access markets: A price war," in In proceedings of GLOBECOM'10, 2010, pp. 1-5. 